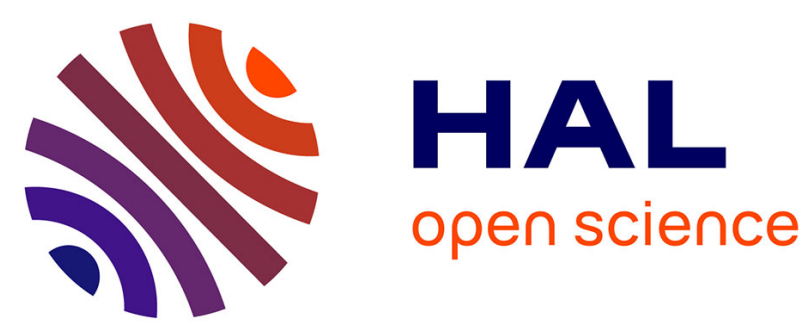

\title{
Elastic properties of continental carbonates: From controlling factors to an applicable model for acoustic-velocity predictions
}

\author{
Jean Baptiste Regnet, Jerome Fortin, Aurélien Nicolas, Matthieu Pellerin, \\ Yves Gueguen
}

\section{To cite this version:}

Jean Baptiste Regnet, Jerome Fortin, Aurélien Nicolas, Matthieu Pellerin, Yves Gueguen. Elastic properties of continental carbonates: From controlling factors to an applicable model for acousticvelocity predictions. Geophysics, 2019, 84 (1), pp.MR45-MR59. 10.1190/geo2017-0344.1. hal02329561

\section{HAL Id: hal-02329561 \\ https://hal.science/hal-02329561}

Submitted on 15 May 2020

HAL is a multi-disciplinary open access archive for the deposit and dissemination of scientific research documents, whether they are published or not. The documents may come from teaching and research institutions in France or abroad, or from public or private research centers.
L'archive ouverte pluridisciplinaire $\mathbf{H A L}$, est destinée au dépôt et à la diffusion de documents scientifiques de niveau recherche, publiés ou non, émanant des établissements d'enseignement et de recherche français ou étrangers, des laboratoires publics ou privés. 
Elastic properties of continental carbonates: from controlling factors to an applicable model for acoustic velocity predictions

(1)

Regnet J.B. ${ }^{{ }^{*}}$, Fortin J. ${ }^{a}$, Nicolas A. ${ }^{a}$, Pellerin M. ${ }^{b}$, Guéguen $Y .{ }^{a}$

a Laboratoire de Géologie de l'Ecole Normale Supérieure - PSL Research University - UMR CNRS 8538, Paris, France

b TOTAL, CSTJF, Avenue Larribeau, Pau, France

* Corresponding author: regnet@geologie.ens.fr, Ecole Normale Supérieure, Laboratoire de Géologie UMR CNRS 8538, 22 rue Lhomond, Paris, France. Now at the department of Civil Engineering and Applied Mechanics, McGill University, Canada.

\section{ABSTRACT}

This study provides new insights into (1) the controlling factors of elastic properties in continental carbonate rocks, and (2) introduces an applicable model for acoustic velocity predictions in such medium. Petrophysical properties (porosity, permeability, P and S-wave velocities...) from laboratory measurements have been coupled with thin section observations and characterizations, and X-Ray Diffraction analyses. A major achievement is the establishment of the link between the mineralogical composition and the $\mathrm{P}$ and $\mathrm{S}$-wave velocity dispersion at a given porosity. This reflects the subtle interplay between physicochemical and biological precipitation of continental carbonates, which can also be associated with a strong influence of detrital mineralogical inputs. The result is a mineralogical commixture, coupled to a wide array of pore types inherited from the strong ability of carbonate rocks to undergo diagenetic alteration. The proposed model takes into account the elastic moduli of the minerals, porosity, and pore shape, and is based on the effective medium 
theory. We consider the case where the medium contains randomly oriented pores with different aspect ratios. Overall, the fit between predicted trends and the experimental data is fairly good, especially for calcite and quartz matrix mineralogy. The results are even better when considering mineralogy inferred from X-Ray Diffraction data, although in some case, and despite the aspect ratio variation in both simulations, the model fails to accurately predict the P-wave velocities. This probably means that another factor is at stake beside mineralogy. This can be explained by the limitation of the effective medium approach which oversimplifies the reality and fails to account for the variability of some aspect ratio from one inclusion to another.

\section{INTRODUCTION}

Carbonate rocks are convoluted and heterogenous media by nature. This inherent complexity is a main concern when deciphering seismic data for subsurface characterization. The relations and correlations between elastic properties and carbonate rock type or rock physical properties (porosity, mineralogy...) is of primary importance to interpret and understand seismic sections and acoustic logs (Anselmetti et al., 2001; Kenter et al., 2007; Regnet et al., 2015a). Only then, the source of reflectivity on seismic lines can be identified and fully understood, as so the parameters used to calculate impedance models for synthetic sections (Anselmetti and Eberli, 1993; Eberli et al., 2003). It is common knowledge that many parameters as the dominant mineralogy, pore type and pore shape, porosity and pore fluid, rock fabric, influence the elastic properties of carbonate rocks in general (among the many publications available, see in particular Eberli et al., 2003; Assefa et al., 2003; Verwer et al., 2008; Weger et al., 2009; Fournier \& Borgomano, 2009; Fournier et al., 2011; Regnet et al., 2015a, 2015b). The attempt to define reliable correlations between all those physical properties becomes a dilemma and sometimes an obstacle when trying to disentangle the seismic or log signature in carbonate succession. 
The discovery of deep and ultra-deep water giant fields in offshore Brazil has put continental carbonate deposits under the spotlight, as they account for a large portion of the production (Bruhn et al., 2003). Yet, the lack of non-marine carbonate reservoirs throughout the geological record means their physical properties are poorly documented and thus poorly understood. Furthermore, the extensive use of seismic imaging or 3D seismic imaging technics and their results on the offshore Brazil deposits are sometime delicate to handle due to the overlying thick sequence of salt ("pre-salt deposits") (Bassant et al., 2005; Thompson et al., 2015). The origin of continental carbonates is a subtile interplay between physicochemical and biological precipitation of carbonates, that can be coupled with a strong influence of clastic mineralogical inputs. The result is a mineralogical commixture, coupled to a wide heterogeneity of pore types usually inherited from the great diagenetic potential of carbonate rocks (from cementation and dissolution to recrystallisation or dolomitization...)

Taking into account this natural complexity, we propose new elements of reflexion on the controlling parameters of elastic properties in continental carbonate rocks, gathered from various localities in the world (outcrops and subsurface). Compressional-wave velocity $\left(\mathrm{V}_{\mathrm{p}}\right)$ and shear-wave velocity $\left(\mathrm{V}_{\mathrm{s}}\right)$ measurements under confining pressure, alongside with porosity, mercury injection measurements, X-Ray Diffraction (XRD) and petrography are used to characterize the samples. Then, based on the experimental results and the insights gained from the controlling factors, we suggest a simple analytical model based on the effective medium theory, for acoustic velocity predictions in those rock media.

\section{METHODOLOGY}

Samples described in this study are assembled from different outcrops and subsurface analogues of pre-salt carbonates. 


\section{COMPOSITION characterization}

Texture, composition and pore space/type were characterized on both thin sections (with luminescent-died epoxy impregnation) and with observations carried out on the macroscopic scale.

X-RAY Diffraction (XRD) analyses were conducted on 40 samples were petrography suggested a complex mineralogical composition. The semi quantitative interpretation is done with Quanta software. For sake of simplicity, only three dominant mineralogical assemblages are discriminated regarding their known effect on the elastic properties: calcite, dolomite, and the quartz-clay assemblage (Anselmetti et al., 1997, Eberli et al., 2003 and Kenter et al., 2007). Grain density of the samples is determined with a Micromeritics Accupyc 1330 helium pycnometer.

\section{PHYSICAL properties}

The petrophysical measurements are conducted on sixty five 1.5 -inch $(3.81 \mathrm{~cm})$ diameter plugs. The effective porosity in the plugs is measured by means of helium expansion porosimetry. Mercury injection porosimetry was performed using an Autopore IV 9500 porosimeter on 20 samples. It can generate pressures from 3.103 to $200 \mathrm{MPa}$, providing access to pores with throat diameters of $360 \mu \mathrm{m}$ to $0.03 \mu \mathrm{m}$. Gas permeability was measured in a steady state permeameter with nitrogen $\left(\mathrm{N}_{2}\right)$.

Compressional-wave and Shear-wave velocities $\left(\mathrm{V}_{\mathrm{p}}\right.$ and $\mathrm{V}_{\mathrm{s}}$ respectively) measurements are performed under dry conditions and at different hydrostatic pressures $(2.5,5,10,20$ and 40 $\mathrm{MPa}$ ) in a loading and unloading path (hysteresis loop). The two wave types propagate along the sample axis at a frequency of $1 \mathrm{MHz}$ (Ultrasonic domain). The measurement accuracy is estimated to be within $3 \%$. The elastic moduli (bulk modulus $\mathrm{K}$, shear modulus $\mathrm{G}, \ldots$ ) are inverted from the velocity measurements and the dry bulk density. 
RESULTS

\section{MICROSTRUCTURES}

Microstructural observations reveal a large variation of sedimentary texture. Samples can be grouped into four texture association based on their composition and structures. (1) Bioclastic grainstone (coquinas) are characterized by an intense calcite cementation, with a non-negligeable overprint of chalcedony (the silicified spots are always several millimeters in cross-section) (Figure 1A \& B). Due to the cementation, the outline of the coquina shells have been obliterated but is still visible in the large calcite crystals. Porosity is uniformly widespread and essentially intercrystalline or moldic, and occurs where the cementation of cavities is uncomplete. Few samples have been microsparitized, revealing a minor microporosity fraction (Figure 1C \& D). (2) Lithoclastic to bioclastic wackestones-packstones contain partially dolomitized lithoclasts of various size (silt-sized to large sand-sized quartz and clay) (Figure 1E). Bioclastic fraction consists of eroded and rounded shell fragments from ostracods to gastropods. The grains are embeded in a calcite-rich matrix, although some small sized blocky calcite cements might occur between them. Most of the porosity is interparticular, corresponding to parts that have not been cemented. Some dolomite cement reduced and biomoldic porosity is also regularly present, as microporosity in the matrix (Figure 1F). (3) Microbial bindstone refers to stromatolite deposits and consist of micritic laminae of algal or microbial origin (framework). Layers alternate from calcite cemented to micritic ones that can contain detrital minerals (mostly quartz and clay, but also feldspars or muscovite) (Figure 2A). Porosity is mostly intercrystalline $(50 \mu \mathrm{m}$ large pores) and some vuggy pores can occur in layers that are coarser crystalline and which rather correspond to solution enlarged veins that have been partially infilled by cement (Figure 2B \& C). Microporosity is not widespread and is limited to micritic intervals, following the laminations 
127 (Figure 2D). (4) Biomicrite shrubs represent travertines schrub samples with arbustiform 128 framework or honey-comb like structures of algal origin (Figure 2E \& F). Grains are mostly small peloids and detrital grains (mainly quartz) that are present all over this rock type and are found along the lamination. Some rhomb shape crystals point to a dolomite mineralogy. Porosiy is mainly vuggy as well as interparticular. Dessication-like cracks regularly occur around few grains, as well as some circular pores. Some of the micritic layers possess welldeveloped microporosity.

\section{MINERALOGICAL composition}

Optical observations reveal a complex mineralogical mixing where calcite, dolomite and detrital minerals (quartz and clay mostly) co-exist. Few samples (18) have a pure mineralogical composition with almost $100 \%$ of calcite or dolomite (Figure 3). Those pure mineralogical composition are associated with bioclastic 'coquina' grainstones (calcite mineralogy) and in a lesser extent, with bioclastic wakestones-packstones (dolomite oriented). The two other rock types (stromatolite laminite and biomicrite schrubs) are strongly affected by detrital quartz or clay inputs (indisolubles in Figure 3) and by dolomite recrystallization.

\section{POROSITY, permeability and mercury injection}

Porosity displays a wide range of values from $1 \%$ to $39 \%$ with a median value of $13 \%$, and permeability data show a cloudy distribution, over several orders of magnitude, between $10^{-3}$ and $4445 \mathrm{mD}$ (Figure 4). As it is often the case in carbonate rocks, the porositypermeability relationship deviates strongly from a clear linear or power-law correlation. When considering the rock-type, Figure 4A shows a large superposition of the porosity-permeability properties regarding the texture, and the permeability range for one texture can be relatively high (4 orders of magnitude). The porosity-permeability evolution trend of Bioclastic 
wakestones-packstones appears to be well-constrained. The influence of dominant pore-type on permeability is not clear even if microporous samples tend to have the lowest values at a given porosity range (Figure 4B). The intercrystalline to moldic porosity (sensu Lucia, 2007) samples display a wide variation of fluid flow properties as the two pore types coexist in different relative proportions respect to one another.

Given the strong diagenetic overprint through solution-enlarged pores, inconsistencies between helium and mercury injection porosity values are expected and observed (Figure 5A). The later ones are largely under-estimated in the high porosity domain (> 20\%). This could show that most of the pore space in high porosity samples is supported by larger pores that are not investigated by mercury injection measurements (such as solution-enlarged interparticular pores, vuggs or moulds). This presence of larger porous inclusions is supported by the derivative of the cumulative intrusion curves with respect to pore-throat diameter (PTD, Figure 5B \& C). They show skewed and unimodal distribution of PTD when Hg-porosity is equivalent to Gas porosity (orange dots and curves, Figure 5A \& B), with clearly one single family of pores and subsequent mercury filling through pores with smaller size. On the opposite, "non-consistent porosity" sample (blue dots and curves, Figure 5A \& C) curves display a plurimodal and wide distribution of PTD, except the one with a rather unimodal and a wide peak distribution (red curve, Figure 5C) which also displays a higher mercury porosity respect to the gas porosity one.

To make sense of those results, and the role played by the pore throat diameter on the fluid path and flow, we perform simple calculations using a permeability model where the PTD value at the main peak is the one that controls the fluid flow. Doing so, we will disregard for the other peaks even if it may not be so accurate as irreversible changes in the microstructure can happen with hydro-mechanical coupling under high stresses. 
Guéguen and Dienes (1989) derived expressions for the permeability based on a statistical model made of interconnected pipes that are located at random points in space, with specific geometrical properties (length and radius). In this medium, the permeability depends on the statistical mean values of the pipe radius and length, and of course, on the pipe density. Summarizing their results, the authors come up with the following relation for the permeability:

$$
K=\frac{1}{32} \phi \bar{r}^{2}
$$

where $\bar{r}$ is the mean pipe radius and $\phi$ is the porosity. Considering here that we deal with peak-like distributions, we take $\bar{r}=d_{c} / 2$ for the mean pipe radius. Here, we assume again that fluid flow is driven by the critical diameter, even if the physical background of the statistical model is very different from a percolation model. This model worked particularly well in other carbonate rocks (Casteleyn et al., 2011). Figure 6 shows a fairly good permeability prediction (orange dots) when porosity measurements are consistent. The PTD is then a good proxy for permeability. When considering other samples, and especially the "nonconsistent porosity" ones (blue dots), calculated permeability is largely under-estimated. This comes from the omission of other pore families in the calculation that are likely to greatly influence the fluid-flow.

\section{ACOUSTIC properties}

High pressure P-wave velocity measurements show that a distinction can be made between velocity paths that remain more less constant and stabilize (terminal velocity) with an increasing pressure (black-dashed cruves, figure 7) and velocity paths that significantly increase with an increasing pressure (red curves, figure 7). This phenomenon can possibly be explained by the presence of microcracks or crack-like pores within the samples, that tend to close during the progressive loading (Fortin et al., 2007; Regnet et al., 2015a, 2015b; Nicolas 
et al., 2016). For the later samples, velocities never stabilize suggesting that $40 \mathrm{MPa}$ is still far from the complete closing pressure $\left(P_{\text {closure }}\right)$ of microcracks. It is noteworthy that the loading pressure-velocity path is steeper than during the release path, resulting in a non-hysteresis loop. This effect shows that the elastic limit has been reached for some of the samples, and that some microcracks, crack-like pores or brittle mechanisms might occur during the loading path. This crack-related effect will be discussed later in the paper.

$\mathrm{P}$ and S-wave velocities range from 2725 to $6377 \mathrm{~m} / \mathrm{s}$ and from 1606 to $3444 \mathrm{~m} / \mathrm{s}$ respectively (Figure 8A). Here, total porosity controls the acoustic velocities as they decrease with increasing porosity. However, velocity dispersion at a given porosity is quite huge and reach $2300 \mathrm{~m} / \mathrm{s}$ for P-waves and $1200 \mathrm{~m} / \mathrm{s}$ for S-waves.

Both bulk (K) and shear moduli $(\mathrm{G})$ follow the same linear trend as acoustic velocities with total porosity (Figure $8 \mathrm{~B}$ ). The scattering of values on $\mathrm{K}$ modulus is more or less $50 \%$, especially for low porosities $(<20 \%)$. Poisson's ratio show no correlation with porosity but a cloudy distribution (Figure 7C). Variations go from 0.11 to 0.36 , with most of the values centered around 0.30 . The grain densities, ranging from 2.63 to $2.85 \mathrm{~g} / \mathrm{cm}^{3}$, but centenred to calcite values $\left(2.70 \mathrm{~g} / \mathrm{cm}^{3}\right.$, Mavko et al., 2009). This result along with observations made on the Poison's ratio goes with the observed complex mineralogical composition of the samples. Here, mineralogies that can push the grain density towards lower values are quartz and clay (2.65 g/cm ${ }^{3}$ and 1.58 to $2.6 \mathrm{~cm}^{3}$, respectively (Mavko et al., 2009). Heavier mineralogies that occur in the samples is dolomite $\left(2.88 \mathrm{~g} / \mathrm{cm}^{3}\right)$. Even within the same mineralogy, the grain density can vary. Carbonates for example are associated with fabric alternations where sparite crystals can be more dense than micritic fabrics (Pentecost, 2005). 
In carbonate rocks, elastic properties are a complex product of several parameters which together control range and absolute values of velocity. In the next sections, we discuss and bring insight into the velocity-controlling factors of continental carbonate rocks, compare their significance, and propose an adequate model to predict them.

\section{FACTORS controlling velocity in continental carbonate rocks}

\section{PRESSURE and cracks}

In many cases, P-wave velocities are known to be pressure and crack-density dependant (Fortin et al., 2007; Regnet et al., 2015a, 2015b; Nicolas et al., 2016). When considering the used isostatic pressure range (2.5 $\mathrm{MPa}$ to $40 \mathrm{MPa})$, velocity dispersion values become more and more centered to zero, as porosity increases (Figure 9A). This marks the diminishing role of cracks in terms of velocity dispersion and more rounded porous inclusions towards high porosity values.

The evolution of acoustic velocities can be interpreted theoretically and quantitatively considering a porous rock as a mixture of solid grains, spherical pores and penny-shaped cracks. By inverting the crack densities, a quantitative description of crack-induced velocity dispersion is then accessible. Here, we understand crack density as a statistical generalization of porosity for non-spherical inclusions. An elongated pore between two grains will be considered a crack (Guéguen \& Kachanov, 2011; Mallet et al., 2013, Ghabezloo, 2015).

Using Kachanov (1993), the effective elastic properties of the porous rock may be expressed as a function of the overall porosity $\boldsymbol{\Phi}$ and the crack density $\boldsymbol{\rho}_{c}$. The crack density is defined as:

$$
\rho_{c}=\frac{1}{V} \sum_{i=1}^{n} c_{i}^{3}
$$

where $\boldsymbol{N}$ is the total number of cracks embedded in the representative elementary volume (REV) $\boldsymbol{V}$ and $\boldsymbol{c}_{\boldsymbol{i}}$ is the radius of the $i$ th crack. One can note that from equation 2, the dominant 
aspect of the crack density is the crack length $c$ and not the number of cracks per unit of volume, which can be non-intuitive.

Following Kachanov (1993) and Fortin et al. (2007), the effective bulk modulus $\boldsymbol{K}$ (in dry conditions) can be expressed as:

$$
\frac{K_{0}}{K}=1+\frac{\rho_{c}}{1-\phi} \frac{h}{1-2 v_{0}}\left(1-\frac{v_{0}}{2}\right)
$$

where $\boldsymbol{K}_{\boldsymbol{0}}$ is the bulk moduli of the crack-free matrix, $\boldsymbol{v}_{\boldsymbol{0}}$ is Poisson's ratio of the crack-free matrix, $\boldsymbol{\rho}_{\boldsymbol{c}}$ is the crack density and $\boldsymbol{h}$ is a factor given (for a penny-shaped geometry) by:

$$
h=\frac{16\left(1-v_{0}^{2}\right)}{9\left(1-v_{0} / 2\right)} \text {. }
$$

The elastic properties $\boldsymbol{K}_{\boldsymbol{0}}$ and $\boldsymbol{v}_{\boldsymbol{0}}$ of the solid matrix and embedded pores were estimated from velocities measured at $\mathrm{Pc}=40 \mathrm{MPa}$, a pressure far below the crack closing pressure for some of the samples (red curves, Figure 7), as the velocities keep building up beyond this point. In other words, this value of $40 \mathrm{MPa}$ should be seen as a lower bound limit of the crack closing pressure for the considered samples, and the calculated crack densities as minimum values. Here, crack density takes values between 0.2 and 0.6 , and mostly close to zero in the high porosity domain (> 20\%, Figure 9B). Also, the samples with the highest crack density value are largely clustered to the main porosity-velocity trend. This means that most of the initial cracks are closed at an isostatic pressure of $40 \mathrm{MPa}$, and that residual cracks that would have been closed beyond $40 \mathrm{MPa}$, should not influence the velocity that much.

Following Walsh (1965), the crack closure pressure $\boldsymbol{P}_{\boldsymbol{c l}}$ for isotropic stress state can be related to the crack aspect ratio defined as $\xi=w / 2 c$, where $\boldsymbol{w}$ is the crack aperture:

$$
P_{c l}=\frac{\pi \xi E_{0}}{4\left(1-v_{0}^{2}\right)}
$$

271 Using the elastic values $\left(\boldsymbol{E}_{\boldsymbol{0}}\right.$ and $\left.\boldsymbol{v}_{\boldsymbol{0}}\right)$ of the crack-free material at $\boldsymbol{P}_{\boldsymbol{c l}}=40 \mathrm{MPa}$ and equation 5 , 272 the minimum crack aspect ratios $\xi$ fall between $5.10^{-4}$ and $4.10^{-3}$, and rise from low to high 
porosity values (Figure 9C). This result is somehow expected as the pore and crack aspect ratios become larger when porosity rises. It is noteworthy that in dry case, crack aspect ratio has little influence on the elastic properties, where they become paramount in saturated conditions due to fluid content/microstructure coupling (local fluid-flow from cracks to pores, also called "squirt-flow", O'Connell and Budiansky, 1977).

Following Guéguen and Kachanov (2011), if cracks are assumed to be characterized by a penny-shape geometry, the crack porosity is given by:

$$
\phi^{\text {crack }}=2 \pi \rho_{c}\langle\xi\rangle,
$$

where $\xi$ is the average crack aspect ratio defined as $\langle\xi\rangle=\langle w / 2 c\rangle$. Taking $\xi$ values and crack density values inverted at $\mathrm{Pc}=40 \mathrm{MPa}$, the initial crack porosities between 0 and $0.4 \%$ (Figure 9D). Finally, crack density or their aspect ratio have limited effect on the elastic properties and should not be taking into account when it comes to the origin of velocity dispersion.

\section{ROCK fabric and pore type}

In carbonate rocks, the depositional settings and diagenetic pathway are known to form special rock fabrics with characteristic velocity patterns. Here, the dataset being an assemblage of samples from various locations, both the depositional setting and the diagenetic evolution is obviously not the same from one sample to another. Thus, an effect of the rockfabric (texture, facies, composition) on the porosity-velocity trend is hardly expected, as shown by Figure 10A. Litho-Bioclastic packstones are rather positioned on a lower porosityvelocity trend, partly due to their detrital mineral fractions (Figure 3).

Several studies have shown that acoustic velocity dispersion in carbonate rocks can be explained by the variability of pore types and geometries (Eberli et al., 2003; Weger et al. 2009). Here, pores are mainly composed of a (1) Intercrystalline-Moldic mixing, and (2) vugs 
(from several tens of $\mathrm{mm}$ to $1 \mathrm{~cm}$ ) and does not explain the entire variability of P-wave velocities, even for the highest porosities (Figure 10B).

\section{MINERALOGY}

The difference between the elastic properties of calcite and dolomite is high enough so that, similar to siliciclastic rocks, some control of the mineralogical composition on the velocity of carbonate rocks might be expected. Total porosity controls the overall elastic response here, but the mineralogical overprint is a good candidate to explain the wide variability of the acoustic wave velocities at a given porosity (Figure 11). The lower porosityvelocity trend is dominated by samples with medium to large quartz/clay proportions $(12 \%$ to $80 \%$ ). The presence of minerals of detrital origin (here, mostly quartz and clay) is diffused in the rock-fabric and thus directly effect the elastic properties. Collectively, this observation is consistent with the results of Anselmetti and Eberli (1993) and Kenter et al. (1997), who show that, for a given porosity, mixed carbonate and siliciclastic minerals ( $>5 \%$ in proportion) have lower velocity than pure carbonate rocks.

On the opposite, dolomitized samples are not only found in the higher trend as one should expect, but also centered in the average trend of the dataset (Figure 11). This show that the effect of dolomitization on the elastic properties is much more diluted, as the associated features of dolomitization also strongly modify the existing pore geometry and the total porosity as well (Anselmetti et al., 1997; Eberli et al., 2003). Note that mixed quartz-clay and dolomite samples are also roughly centered on the average porosity-velocity trend. Then, although the mineralogical effect on elastic properties is strong and clear for calcitesiliciclastic mixing, it becomes reduced when dolomitization occurs. In this case, and despite the differences in porosity, elastic properties might also depend on the pore geometry as reported in a near-pure calcite travertine succession where stiff inclusions are dominant (Soete 
et al., 2015) (Figure 11). Linking elastic properties to anyone parameters becomes then impossible as the velocity signal is averaged, and the dataset being composed of samples with rather different diagenetic pathways and evolution.

When time comes to extrapolate those results to other continental carbonate successions or units, not much material is available. Yet, in the literature, the clastic mineralogical mixing is somehow very common: this can be easely explain, as the sources for detritic input are high in those type of sedimentary systems (Soete et al.,2015; Thompson et al., 2015; Ronchi et Cruciani, 2015; Teboul et al., 2016). This means that the highlighted control of the mineralogy, and especially the siliciclastic mixing, on the elastic properties could be globalised and extended to other continental carbonate systems. Yet, the lack of non-marine carbonate reservoirs throughout the geological record means their physical properties are poorly documented and thus poorly understood. The long-term objective is to find modernday analogues to further increase our understanding and knowledge of continental carbonates, driven by the concept that "the present is the key to the past" (Thompson et al., 2015). formulations for bulk moduli but yet sophisticated enough to take into account the elastic moduli of the minerals, porosity, and pore shape. There are several approximate schemes to do this, commonly known as effective medium theory. Among them, the differential effective medium theory proposed by McLaughlin, 1977, Norris, 1985, Salganik, 1973 and Zimmerman, 1984, calculates the effective elastic moduli when inclusions are introduced into the medium in small amounts. The effective moduli is then re-calculated at each step, leading to a pair of differential equations for the effective bulk and shear moduli. Those predictions 
(1961). Even at high inclusion concentrations, this approach produce accurate results

349 (Saenger, Kruger, \& Shapiro, 2004, 2006; Carvalho \& Labuz,c1996; Zimmerman, 1991a;

David et al., 2011). Here, we propose to use asymptotic expressions for both compressibility

351

352

353

354

355

356

357

358

$$
\left(\frac{-3}{4 \pi \alpha}\right) \ln \left(1-\frac{4}{3} \pi \alpha \rho_{c}\right)=-\frac{5}{8} \ln \left(\frac{v}{v_{0}}\right)+\left[c_{1} \ln \left(\frac{v}{v_{0}}\right)+c_{2}\left(\frac{1}{v}-\frac{1}{v_{0}}\right)\right]
$$

$+\alpha^{2}\left[c_{3} \ln \left(\frac{v}{v_{0}}\right)+c_{4}\left(\frac{1}{v}-\frac{1}{v_{0}}\right)+c_{5}\left(\frac{1}{v^{2}}-\frac{1}{v_{0}^{2}}\right)\right]$,

$\left(\frac{K}{K_{0}}\right)=\left[\frac{10}{9} \ln \left(\frac{v}{v_{0}}\right)-\ln \left(\frac{1-2 v}{1-2 v_{0}}\right)\right]+\alpha\left[C_{1} \ln \left(\frac{v}{v_{0}}\right)+C_{2}\left(\frac{1}{v}-\frac{1}{v_{0}}\right)\right]$

$+\alpha^{2}\left[C_{3} \ln \left(\frac{v}{v_{0}}\right)+C_{4}\left(\frac{1}{v}-\frac{1}{v_{0}}\right)+C_{5}\left(\frac{1}{v^{2}}-\frac{1}{v_{0}^{2}}\right)\right]$,

359 where $\boldsymbol{\alpha}$ is the pore aspect ratio, $\boldsymbol{\rho}_{\boldsymbol{c}}$ the crack/inclusion density, $\boldsymbol{v}$ the Poisson's ratio of the

361 modulus and $\boldsymbol{K}_{\boldsymbol{0}}$ the matrix bulk modulus without inclusions, and

$$
\begin{aligned}
& c_{1}=\frac{25}{864}\left(\frac{48}{\pi}+\pi\right) \approx 0.533, \\
& c_{2}=\frac{5}{288}\left(\frac{48}{\pi}+5 \pi\right) \approx 0.538,
\end{aligned}
$$

362

$$
c_{3}=\frac{5}{81}\left(\frac{82}{\pi^{2}}-\frac{163}{16}+\frac{1753}{1536} \pi\right) \approx 0.579 \text {, }
$$

$c_{4}=\frac{5}{27}\left(\frac{8}{\pi^{2}}-\frac{61}{6}+\frac{137}{768} \pi^{2}\right) \approx-1.407$,

$c_{5}=\frac{5}{9}\left(\frac{1}{\pi}+\frac{5 \pi}{48}\right)^{2} \approx 0.232$. 


$$
\begin{aligned}
& C_{1}=\frac{5}{972}\left(\frac{96}{\pi}+77 \pi\right) \approx 1.408, \\
& C_{2}=-\frac{5}{162}\left(\frac{48}{\pi}+5 \pi\right) \approx-0.956,
\end{aligned}
$$

$C_{3}=\frac{5}{243}\left(\frac{863}{288} \pi^{2}-64+\frac{320}{3 \pi^{2}}\right) \approx-0.486$,

$$
C_{4}=\frac{5}{243}\left(-\frac{47 \pi^{2}}{8}+\frac{341}{3}-\frac{320}{\pi^{2}}\right) \approx 0.479 \text {, }
$$$$
C_{5}=-\frac{5}{11664}\left(\frac{48}{\pi}+5 \pi\right)^{2} \approx-0.412 \text {. }
$$

365 The crack density is related to the total porosity $\boldsymbol{\Phi}$ by

$$
\phi=\frac{4}{3} \pi \alpha \rho_{\mathrm{c}}
$$

The volume occupied by cracks is small, but their compliances can be quite large, since they are inversely proportional to their aspect ratio. To obtain inverted P-wave velocities, we first calculate Poisson's ratio $v$ of the sample in eq. 7 , and then injecting the result in eq. 8 to obtain the $\boldsymbol{K} / \boldsymbol{K}_{\boldsymbol{0}}$ term.

As the key controlling parameter on elastic properties is the mineralogical composition (MC) of the sample, we first ran the simulations considering the three dominant $\mathrm{MC}$ (dolomite, calcite and quartz, see Table 1 for detailed values). We consider the case where the medium contains randomly oriented pores with aspect ratios between $10^{-2}$ (crack-like pores) and 0.4 (oblate spheroids). Keep in mind that theoretically, changing the value of $\boldsymbol{\alpha}$ involves changing the overall porosity and crack/inclusion density. The effect of the later on the elastic properties becomes dominant in low pore aspect ratio domains (eq. 11). Yet, this crack/inclusion density is allowed to vary from 0 to 2 . The porosity varies from $0 \%$ to $40 \%$, in the same range that the experimental data. Results of simulations are shown in Figure 12, and main trends are compared to experimental data. When porosity is zero, predicted P-wave velocities correspond to those found and measured in pure dolomite (Figure 12A), calcite 
(Figure 12B) or quartz (Figure 12C) and depend on used values of $\boldsymbol{K}_{\boldsymbol{0}}$ and $\boldsymbol{v}_{\boldsymbol{0}}$ respectively. For all the three MC, the model logically predicts a decrease of P-wave velocities as porosity increases and we found the usual concave-upward evolution. The role of the pore aspect ratio and their associated compressibility differences and tendencies is also well captured. When the aspect ratio value decreases, the pores become more compliable to dynamic deformation as the wave propagates trough the rock. The first decreasing steps, from $\boldsymbol{\alpha}=0.4$ to $\boldsymbol{\alpha}=0.3$ when considering dolomite and calcite MC slightly shift the porosity-velocity trend downward $\left(\Delta_{\mathrm{vp}}<500 \mathrm{~m} / \mathrm{s}\right.$ at a given porosity value). This observation especially stands for the dolomite and calcite MC, and slightly differs when considering a quartz composition where the porosity-velocity relationship trend is lowered during the first changes in pore aspect ratio. In dolomite and calcite $\mathrm{MC}$, the trend shifting through lower P-wave velocities drastically increases when the pore aspect ratio takes values below 0.3 , and drops to its lower value when $\boldsymbol{\alpha}=10^{-2}$. The crack/pore density value increases and becomes paramount at low $\boldsymbol{\alpha}$ where we observed the higher velocity drops (from $6500 \mathrm{~m} / \mathrm{s}$ to $2000 \mathrm{~m} / \mathrm{s}$ with only a $5 \%$ variation in total porosity with $\alpha=10^{-2}$, Figure 12B). Overall, the fit between predicted trends and the experimental data is fairly good, especially for calcite and quartz matrix mineralogy, and considering aspect ratios between $\alpha=0.35$ and $\boldsymbol{\alpha}=0.15$. (Figure 12B \& C).

To go further, we then predict the P-wave velocity of each sample using their associated porosity, and elastic parameters calculated and inferred from the mean mineralogical composition (XRD data). As an example, the matrix bulk modulus $\boldsymbol{K}_{\boldsymbol{0}}$ of a sample with $60 \%$ of calcite, $30 \%$ of dolomite and $10 \%$ of quartz is considered to be the harmonic mean of each mineral bulk value, respectively. The comparison between predicted and measured velocities are presented in Figure 13A \& B, for two distinct aspect ratio values. Dispersion is calculated in absolute value and is the normalized difference between predicted and measured velocities, 
in percent. When $\boldsymbol{\alpha}=0.15$, the P-wave prediction is fairly good with an average dispersion of $13.6 \%$ (Figure 13A). This dispersion beyond the average (green-dashed line) is lower for porosity below $15 \%$, and increases for highest values where $\mathrm{P}$-wave velocities are almost constantly under-estimated. When increasing the aspect ratio to a value of 0.3 , the average dispersion is equivalent (14.1\%, Figure 13B) but is rather constant through porosity changes. The resulting predicted trend is also very similar to the experimental one, and becomes even closer to it in high porosity domain, where dispersion slightly decreases. Finally, it appears that the best prediction is a combination of two aspect ratio values where $\alpha=0.15$ when porosity is below $15 \%$ and $\boldsymbol{\alpha}=0.3$ for higher porosity (Figure 14). This somehow arbitrary cut-off in the $\boldsymbol{\alpha}$ value gives the best result in terms of dispersion which decreases to $9 \%$ when considering low porosities $(<15 \%)$.

In some cases, and despite the aspect ratio variation in both simulations, the model fails to accurately (beyond the average value) predict the P-wave velocities. This probably means that another factor is at stake beside mineralogy. The concerned samples do not belong to any particular texture association, but instead often correspond to samples with a large proportion of vuggy pores or characterized by rather "rounded" biomoldic pores (Figure $14 \& 15$ ). Those two kind of porous inclusions are clearly an issue for the model, which fails to capture their "positive" feedbacks on the elastic properties, where crack-like pores have "negative" effects. This can be easily explained by the limitation of the effective medium approach which oversimplifies the reality by failing to account for the intrinsic variability of the pore aspect ratio from one inclusion to another. 
The objectives here were (1) to propose new insights into the factors controlling elastic properties in continental carbonate rocks, and (2) to suggest a simple analytical model based on the effective medium theory, for acoustic velocity predictions in those media.

(1) Total porosity controls the overall elastic response here, but the mineralogical overprint is a good candidate to explain the wide variability of the acoustic wave velocities at a given porosity. The presence of minerals of detrital origin is diffused in the rock-fabric and thus directly effects the elastic properties. This observation is also in good agreement with previous studies on the matter. On the other hand, the effect of dolomitization on the elastic properties is much more diluted, as the associated features of dolomitization also strongly modify the existing pore geometry and the total porosity. When trying to extrapolate those results to other continental carbonate successions or units, not much material is available. Yet, in the literature, the clastic mineralogical mixing is somehow very common. This can be easily explain, as the sources for detritic input are high in those type of sedimentary systems. This means that the highlighted control of the mineralogy, and especially the siliciclastic mixing, could be expected and extended to other continental carbonate systems.

(2) The proposed model, based on the elastic moduli of the minerals, porosity and pore shape, is working fairly well when we consider the case where the medium contains randomly oriented pores with aspect ratios between $10^{-2}$ (crack-like pores) and 0.4 (oblate spheroids). Overall, the fit between predicted trends and the experimental data is fairly well, especially for calcite and quartz matrix mineralogy, and considering aspect ratios between $\alpha=0.35$ and $\alpha=0.15$. In some case, and despite the aspect ratio variation in both simulations, the model fails to accurately (beyond the average value) predict the P-wave velocities. This probably means that another factor is at stake beside mineralogy. This can be easily explained by the limitation of the effective medium approach which oversimplifies the reality by failing to account for the intrinsic variability of the pore aspect ratio from one inclusion to another. The 
concerned samples do not belong to any particular texture association, but instead often correspond to samples with a large proportion of vuggy pores or characterized by rather "rounded" biomoldic pores.

\section{APPENDIX A}

Supporting information table containing the petrophysical data presented in this article. Table A1 is organized by sample name with one column per petrophysical properties and derived parameter from our laboratory measurements.

\section{ACKNOWLEDGMENT}

This work is part of a Postdoctoral Fellowship funded by TOTAL R\&D Carbonate Team. Authors are very grateful to Rudy Swennen and the KU Leuven team for carrying the petrographical (thin-section analysis), mineralogical (XRD) and petrophysical analysis. With regard to the latter, density analysis was carried out at KU Leuven, while poroperm analysis was done at Panterra BV (the Netherlands) and the Vp \& Vs measurements were performed at the VU Amsterdam.

\section{1, 287-323.}

Anselmetti, F.S., and Eberli, G.P., 1993. Controls on sonic velocity in carbonates. PAGEOPH

Anselmetti, F.S., and Eberli, G.P., 1999. The Velocity-Deviation Log: A Tool to Predict Pore Type and Permeability Trends in Carbonate Drill Holes from Sonic and Porosity or Density Logs. AAPG Bulletin 83, 450-466.

Assefa, S., McCann, C., and Sothcott, J., 2003. Velocities of compressional and shear waves in limestones. Geophysical Prospecting 51, 1-13.

Bassant, P., Van Buchem, F. S. P., Strasser, A., \& Görür, N., 2005. The stratigraphic architecture and evolution of the Burdigalian carbonate-siliciclastic sedimentary systems of the Mut Basin, Turkey. Sedimentary Geology, 173, 187-232. 
Bruhn, C. H., Gomes, J. A. T., Del Lucchese Jr, C., \& Johann, P. R., 2003. Campos basin: reservoir characterization and management - Historical overview and future challenges. Presented at: Offshore Technology Conference.

Campbell, A.E., and Stafleu, J., 1992. Seismic Modeling of an Early Jurassic, Drowned Carbonate Platform: Djebel Bou Dahar, High Atlas, Morocco. AAPG Bulletin 76, 17601777.

Carvalho, F. C. S., \& Labuz, J. F., 1996. Experiments on effective elastic modulus of twodimensional solids with cracks and holes. International Journal of Solids and Structures, 33, 4119-4130.

David, E. C., \& Zimmerman, R. W., 2011. Elastic moduli of solids containing spheroidal pores. International Journal of Engineering Science, 49, 544-560.

Eberli, G., Baechle, G., Anselmetti, F., and Incze, M., 2003. Factors controlling elastic properties in carbonate sediments and rocks. The Leading Edge 22, 654-660.

Fortin, J., Guéguen, Y., Schubnel, A., 2007. Effects of pore collapse and grain crushing on ultrasonic velocities and Vp/Vs. Journal of Geophysical Research: Solid Earth, 112.

Fournier, F., and Borgomano, J., 2009. Critical porosity and elastic properties of microporous mixed carbonate-siliciclastic rocks. Geophysics 74, E93-E109.

Fournier, F., Leonide, P., Biscarrat, K., Gallois, A., Borgomano, J., and Foubert, A., 2011.

Elastic properties of microporous cemented grainstones. Geophysics 76, E211-E226.

Ghabezloo, S., 2015. A micromechanical model for the effective compressibility of sandstones. European Journal of Mechanics-A/Solids, 51, 140-153.

Guéguen, Y., \& Kachanov, M., 2011. Effective elastic properties of cracked rocks-An overview, in Leroy, Y. M., \& Lehner, F. K., 2011. Mechanics of Crustal Rocks (Vol. 533). Springer Science \& Business Media, 73-126.

Hashin, Z., \& Shtrikman, S., 1961. Note on a variational approach to the theory of composite elastic materials. Journal of The Franklin Institute, 271, 336-341.

Kachanov, M., 1993. Elastic solids with many cracks and related problems. Advances in applied mechanics, 30, 259-445.

Kenter, J., Podladchikov, F., Reinders, M., Van der Gaast, S., Fouke, B., and Sonnenfeld, M., 1997. Parameters controlling sonic velocities in a mixed carbonate-siliciclastics Permian shelf-margin (upper San Andres formation, Last Chance Canyon, New Mexico). Geophysics 62, 505-520.

Kenter, J., Braaksma, H., Verwer, K., and van Lanen, X., 2007. Acoustic behavior of sedimentary rocks: Geologic properties versus Poisson's ratios. The Leading Edge 26, 436444.

Lucia, F. J., 2007. Carbonate reservoir characterization : An integrated approach. Springer, 
2nd edition.

Mallet, C., Fortin, J., Guéguen, Y., \& Bouyer, F., 2013. Effective Elastic Properties of Cracked Solids: An Experimental Investigation. International journal of fracture, 182.

Mavko, G., Mukerji, T., and Dvorkin, J., 2009. The Rock Physics Handbook: Tools for Seismic Analysis of Porous Media (Cambridge University Press).

McLaughlin, R., 1977. A study of the differential scheme for composite materials. International Journal of Engineering Science, 15, 237-244.

Nicolas, A., Fortin, J., Regnet, J. B., Dimanov, A., \& Guéguen, Y., 2016. Brittle and semibrittle behaviours of a carbonate rock: influence of water and temperature. Geophysical Journal International, 206, 438-456.

Norris, A. N., 1985. A differential scheme for the effective moduli of composites. Mechanics of Materials, 4, 1-16.

O’Connell, R.J., and Budiansky, B., 1977. Viscoelastic properties of fluid-saturated cracked solids. Journal of Geophysical Research, 82, 5719-5735.

Pentecost, A., 2005. Travertine. Springer Science \& Business Media.

Regnet, J. B., Robion, P., David, C., Fortin, J., Brigaud, B., \& Yven, B., 2015a. Acoustic and reservoir properties of microporous carbonate rocks: implication of micrite particle size and morphology. Journal of Geophysical Research: Solid Earth, 120, 790-811.

Regnet, J., David, C., Fortin, J., Robion, P., Makhloufi, Y. \& Collin, P., 2015b. Influence of microporosity distribution on the mechanical behavior of oolithic carbonate rocks, Geomechanics for Energy and The Environment, 3, 11-23.

Ronchi, P., \& Cruciani, F., 2015. Continental carbonates as a hydrocarbon reservoir, an analog case study from the travertine of Saturnia, Italy. AAPG Bulletin, 99, 711-734.

Saenger, E. H., Kruger, O. S., \& Shapiro, S. A., 2006. Effective elastic properties of fractured rocks: Dynamic vs. static considerations. International Journal of Fracture, 139, 569-576.

Salganik, R. L., 1973. Mechanics of bodies with many cracks. Mechanics of Solids, 8, 135143.

Soete, J., Kleipool, L. M., Claes, H., Claes, S., Hamaekers, H., Kele, S., ... \& Swennen, R., 2015. Acoustic properties in travertines and their relation to porosity and pore types. Marine and Petroleum Geology, 59, 320-335.

Teboul, P. A., Durlet, C., Gaucher, E. C., Virgone, A., Girard, J. P., Curie, J., ... \& Camoin, G. F., 2016. Origins of elements building travertine and tufa: New perspectives provided by isotopic and geochemical tracers. Sedimentary Geology, 334, 97-114. 
Thompson, D. L., Stilwell, J. D., \& Hall, M., 2015. Lacustrine carbonate reservoirs from Early Cretaceous rift lakes of Western Gondwana: Pre-salt coquinas of Brazil and West Africa. Gondwana Research, 28, 26-51.

Verwer, K., Braaksma, H., and Kenter, J., 2008. Acoustic properties of carbonates: Effects of rock texture and implications for fluid substitution. Geophysics 73, B51-B65.

Walsh, J. B., 1965. The effect of cracks on the compressibility of rock. Journal of Geophysical Research, 70, 381-389.

Weger, R.J., Eberli, G.P., Baechle, G.T., Massaferro, J.L., and Sun, Y.-F., 2009. Quantification of pore structure and its effect on sonic velocity and permeability in carbonates. AAPG Bulletin 93, 1297-1317.

Zimmerman, R. W., 1984. Elastic moduli of a solid with spherical pores: New self-consistent method. International Journal of Rock Mechanics and Mining Sciences, 21, 339-343.

Zimmerman, R. W., 1990. Compressibility of sandstones (Vol. 29). Elsevier.

\section{FIGURE CAPTION}

Figure 1 : Pre-salt analogue samples A, B, C \& D - Coquina grainstones characterized by large, intensely recrystallized coquina shells $(C q ., \mathrm{A}, \mathrm{B}, \mathrm{C} \& \mathrm{D})$. Porosity is mostly interparticular $(P$., picture A \& B), with few microporosity (black and white arrows, $\mathrm{C} \& \mathrm{D})$. Non-negligeable proportion of chalcedony (Ch., A \& B). E \& F - Lithoclastic to bioclastic wackestone-packstone, characterized by partially dolomitised bioclasts $(B c$., $\mathrm{E})$, quartz grains (white arrows, E) and clay $(\mathrm{Cl}$., E). Porosiy is interpaticular and biomoldic (yellow-ish areas and $P .$, F).

Figure 2 : Pre-salt analogue samples A, B, C \& D - Microbial bindstone showing micriticlaminae of algal or microbial origin. Porosity is mainly intercrystalline (Yellow-ish areas is B and C), with localised microporosity in some laminae, following lamination (green luminescent, D). E \& F - Biomicrite schrubs characterized by arbustiform porosity framework with mainly large vuggy pores $(P ., \mathrm{E} \& \mathrm{~F})$. Rhomb shape crystals point to a dolomite mineralogy (white arrows, F).

Figure 3 : Ternplot of mineralogical compositions inferred from XRD measurements. Only the three dominant mineralogical assemblages are considered. The indisolubles contain both quartz and clay mineralogies. Samples are discriminated regarding their microstructural association.

Figure 4 : Porosity-Permeabiliy properties of the pre-salt analogues. Permeability data show a skewed distribution, over several orders of magnitude. This relationship deviates strongly from a clear correlation. A - Data points are discriminated by textures. B - Influence of dominant Pore Types. 
Figure 5 : A - Porosity measurement consistency, between helium and mercury injection. The later ones are almost constantly under-estimated. B \& C - Pore throat diameter extracted from mercury injection measurements. Orange and blue color match with the associated dots in figure A.

Figure 6 : Permeability calculation using a statistical model of interconnected pipes, randomly located in space (Guéguen \& Dienes, 1989). Data points are discriminated regarding measurements consistency of porosity (orange and blue, from Figure 5).

Figure 7 : P-wave velocity paths (loading and unloading) of the high pressure measurements, between $2.5 \mathrm{MPa}$ and $40 \mathrm{MPa}$. Black-dashed and red curves indicate the velocity stability or increase with presure, respectively. Note that the hysteresis loop is not perfect between loading and unloading cycle.

Figure 8: A - P \& S-wave velocity evolution with porosity at $40 \mathrm{MPa}$. B - Bulk and Shear modulii evolution with porosity inverted from velocities at $40 \mathrm{MPa}$. C - Poisson's ratio evolution with porosity inverted from velocities at $40 \mathrm{MPa}$. D - Bulk and Shear modulii evolution at $40 \mathrm{MPa}$ with measured grain density.

Figure 9 : A - Evolution of normalized P \& S-wave velocity dispersion with porosity at 40 MPa. B - P-wave velocity evolution with porosity. Data are discriminated with crack density value inverted from velocities between 2.5 and $40 \mathrm{MPa}$. C - P-wave velocity evolution with porosity. Data are discriminated with crack aspect ratio value closed at $40 \mathrm{MPa}$. D - P-wave velocity evolution with porosity. Data are discriminated with initial crack porosity value.

Figure 10: A - Influence of texture association on the scattering of $\mathrm{P}$-wave velocities at 40 $\mathrm{MPa}$. B - Influence of pore type on the scattering of P-wave velocities at $40 \mathrm{MPa}$.

Figure 11 : P-wave velocity evolution through porosity variation. Data points are discriminated with their quartz/clay content (dot color, associated with the side colobar), and with their dolomite content (dot size). Two mineralogical domains are individualized (calcitedolomite mixing and calcite-quartz mixing zones). Evolution trend collected from Soete et al., 2015 showing velocity-porosity relationship where stiff pore inclusions are dominant is also reported in the figure.

Figure 12 : Analytical simulation of P-wave velocity using the crack-like pores model from David et al., 2011. Simulations are done on three different matrix mineralogies (A - dolomite, $\mathrm{B}$ - calcite and $\mathrm{C}$ - quartz) for a wide range of aspect ratio values from $\alpha=10-2$ to $\alpha=0.34$.

Figure 13 : Analytical simulation of P-wave velocity for two fixed aspect ratio values (A - $\alpha$ $=0.15$ and $\mathrm{B}-\alpha=0.3$ ). Simulations take into account the porosity and the mean mineralogy inferred from XRD analysis, for each sample. Dispersion is the normalized difference between prediction and measurements. 
675 Figure 14 : Analytical simulation of P-wave velocity for two fixed aspect ratio values $(\alpha=$ 6760.15 between $0 \%$ and $15 \%$ porosity, and $\alpha=0.3$ beyond $15 \%$ ). Simulations take into account 677 the porosity and the mean mineralogy inferred from XRD analysis, for each sample. 678 Dispersion is the normalized difference between prediction and measurements. White dots are 679 sample characterized by vuggy and « rounded » biomoldic porosity.

680

681 Figure 15 : Example of samples characterized by high dispersion value regardless the aspect 682 ratio cut-off (white dots, figure 13). A \& B - Bioclastic wackestone-packstone with rather 683 rounded, stiff pores of moldic origin. C \& D - Biomicrite schrubs displaying large vuggy684 frame pores. 

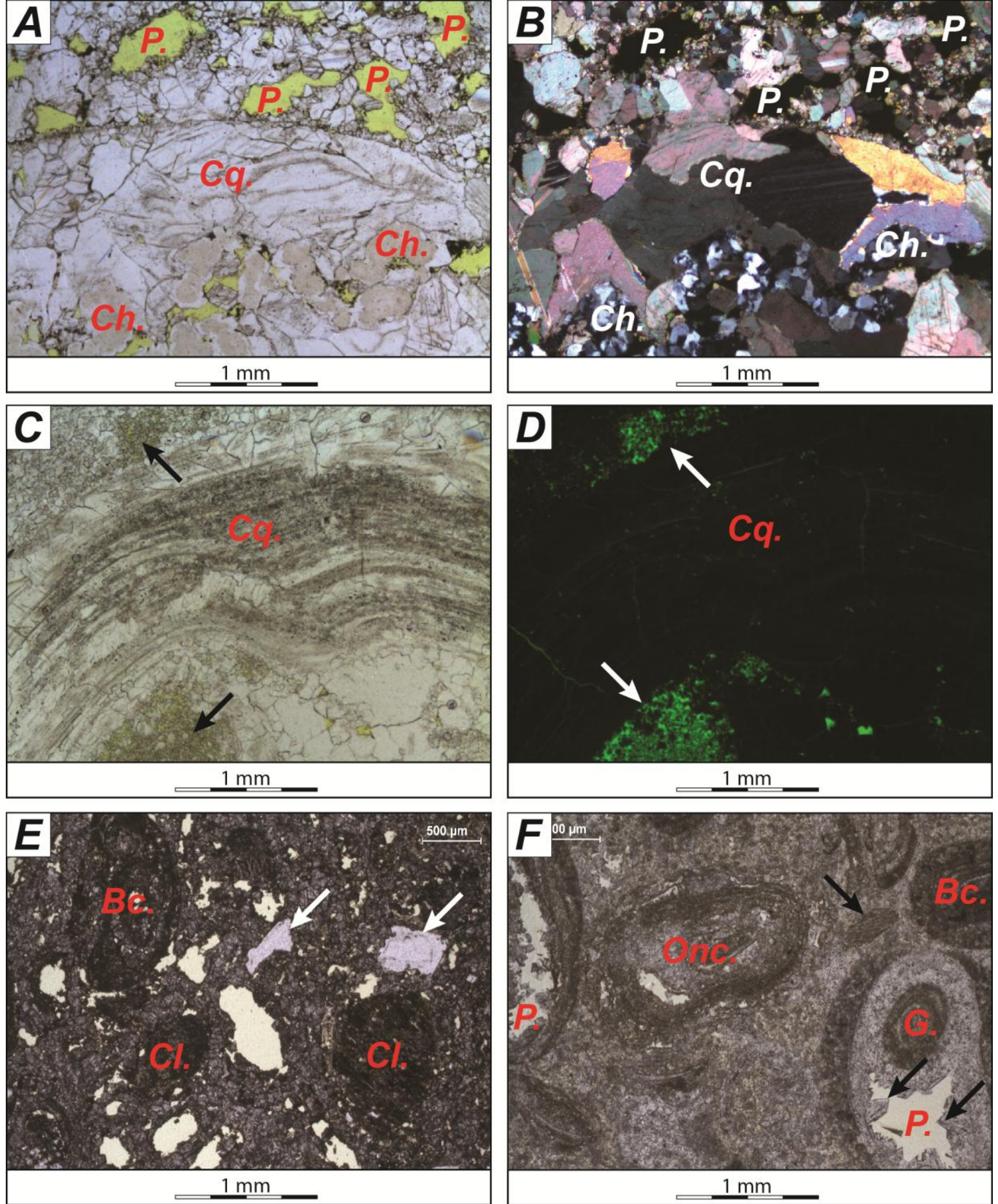

Figure 1 : Pre-salt analogue samples A, B, C \& D - Coquina grainstones characterized by large, intensely recrystallized coquina shells (Cq., A, B, $C \quad \& \quad D)$. Porosity is mostly interparticular ( $P$., picture $\mathrm{A} \& \mathrm{~B})$, with few microporosity (black and white arrows, $C$ \& D). Nonnegligeable proportion of chalcedony (Ch., A \& B). E \& F - Lithoclastic to bioclastic wackestone-packstone, characterized by partially dolomitised bioclasts $(B C ., E)$, quartz grains (white arrows, E) and clay (Cl., E). Porosiy is interpaticular and biomoldic (yellowish areas and $P ., F$ ). 


\section{Indisolubles}

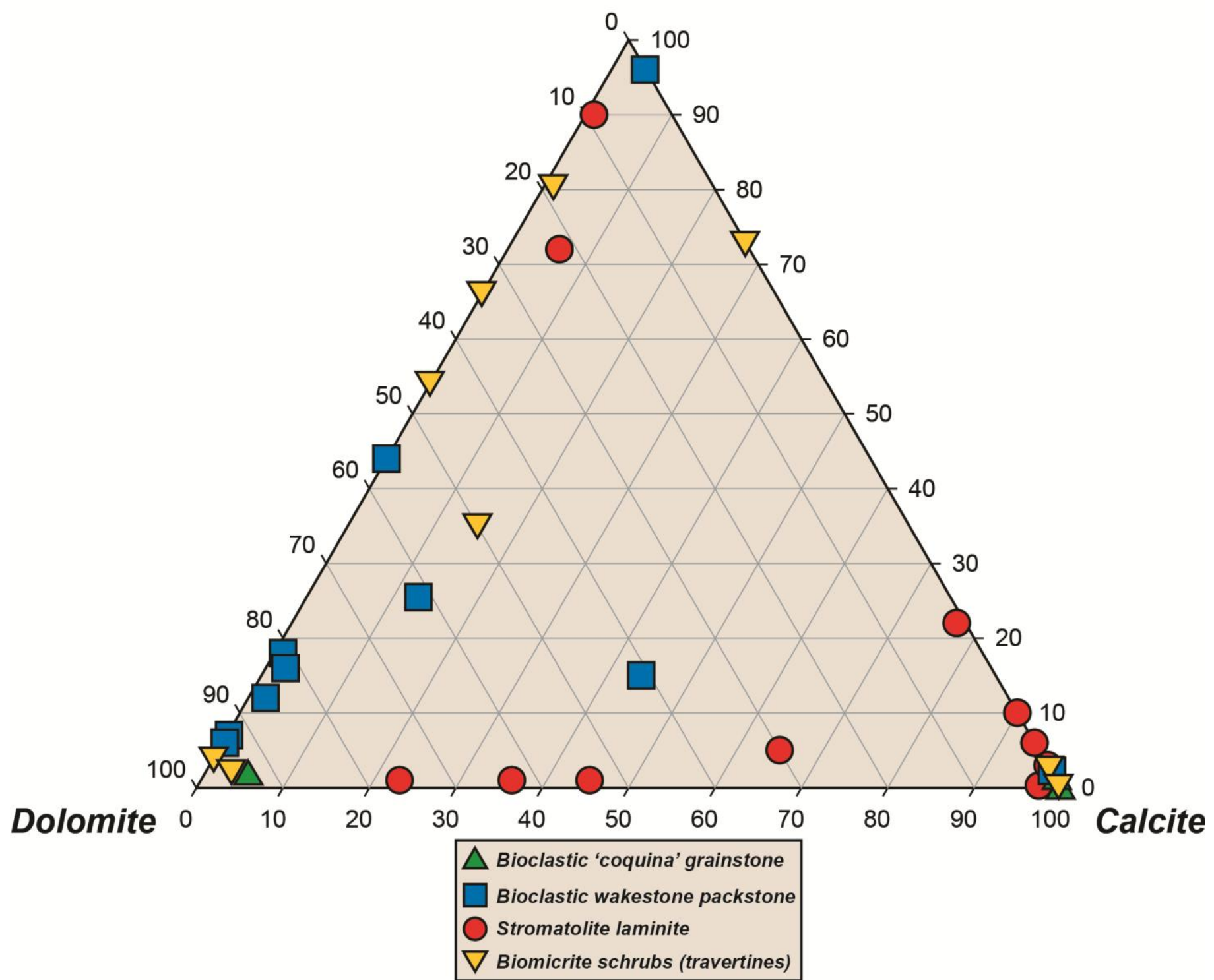

Figure 3 : Ternplot of mineralogical compositions inferred from XRD measurements. Only the three dominant mineralogical assemblages are considered. The indisolubles contain both quartz and clay mineralogies. Samples are discriminated regarding their microstructural association. 

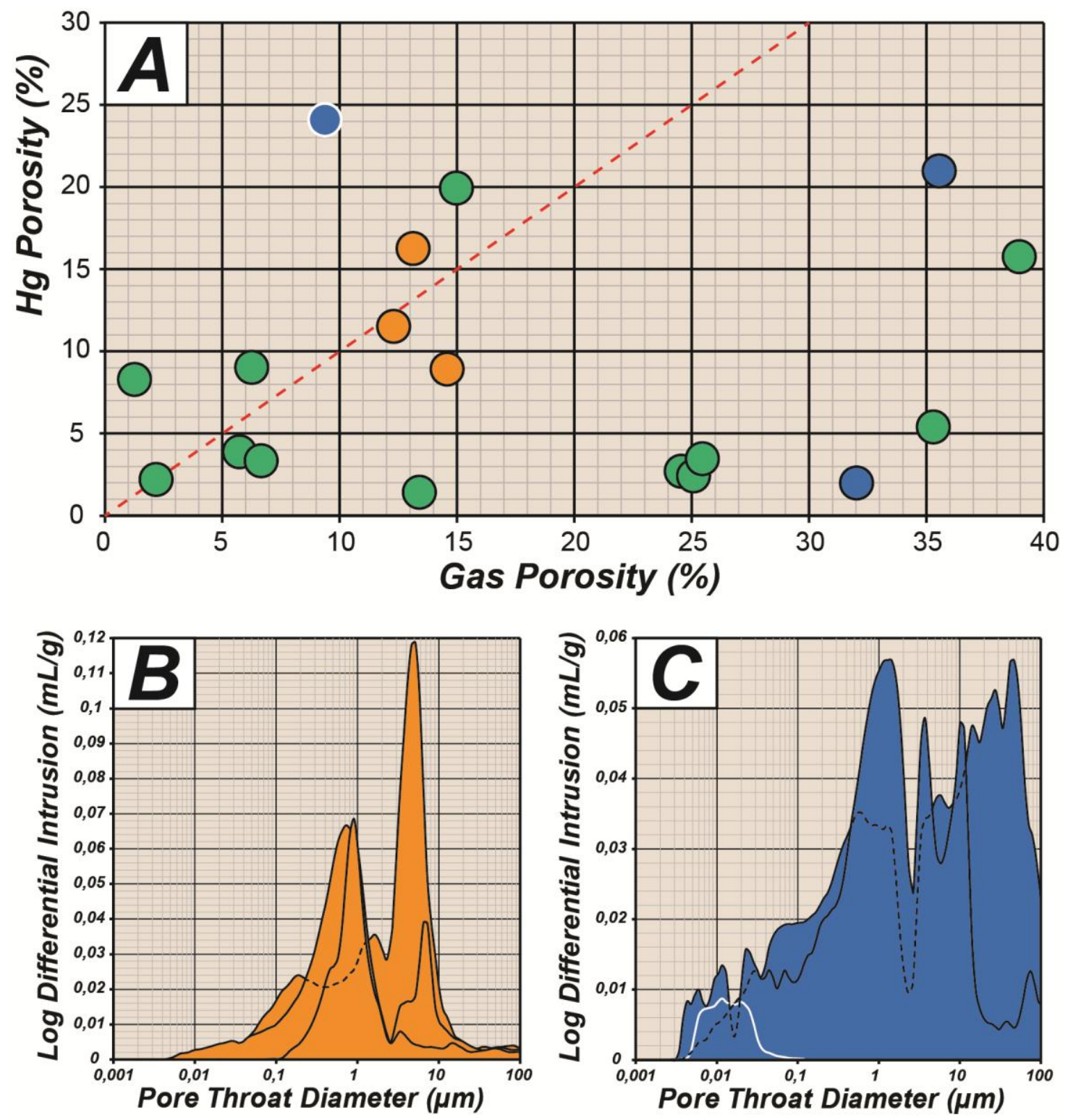

Figure 5 : A - Porosity measurement consistency, between helium and mercury injection. The later ones are almost constantly under-estimated. B \& C - Pore throat diameter extracted from mercury injection measurements. Orange and blue color match with the associated dots in figure $A$. 


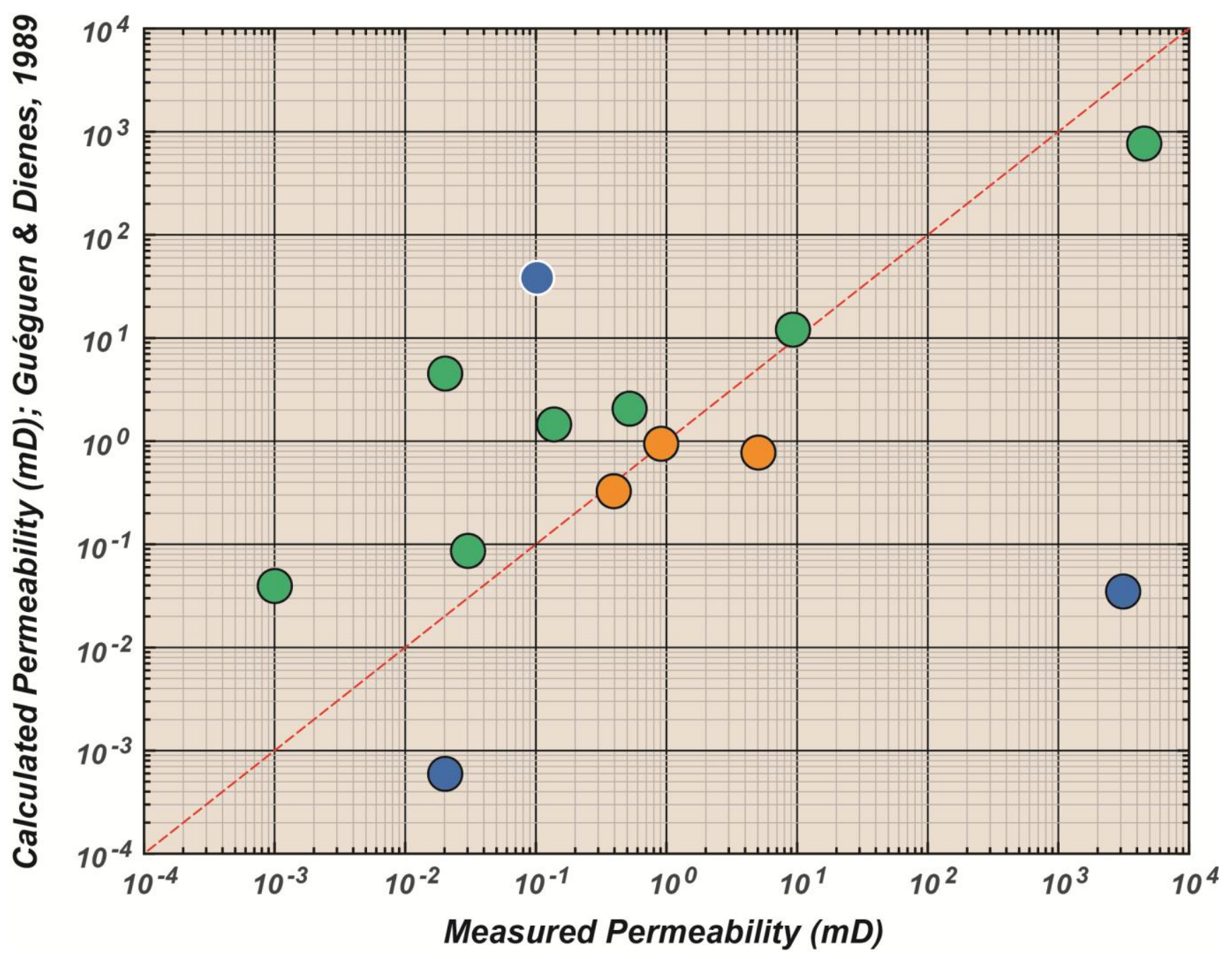

Figure 6 : Permeability calculation using a statistical model of interconnected pipes, randomly located in space (Guéguen \& Dienes, 1989). Data points are discriminated regarding measurements consistency of porosity (orange and blue, from Figure 5). 


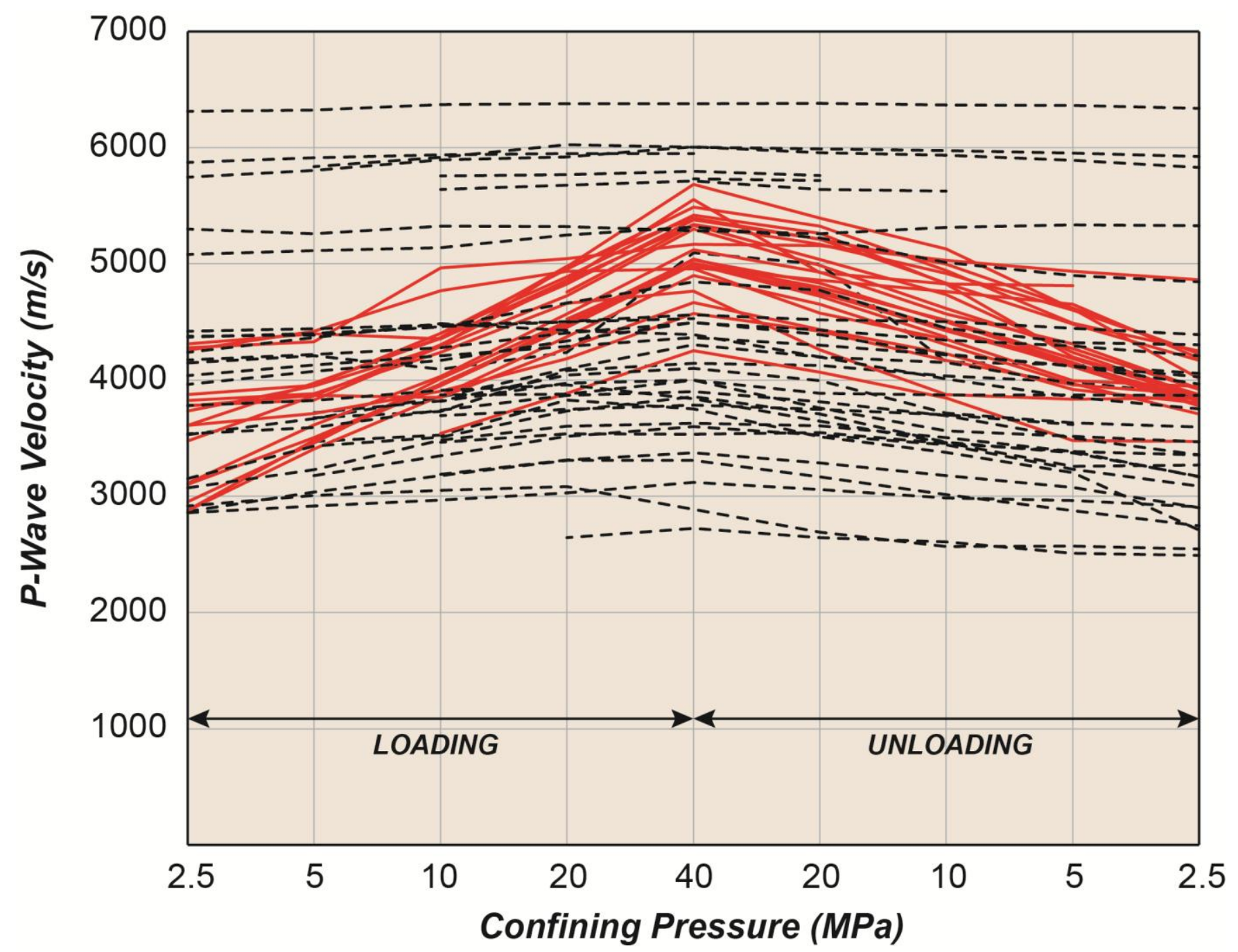

Figure 7 : P-wave velocity paths (loading and unloading) of the high pressure measurements, between 2.5 MPa and $40 \mathrm{MPa}$. Black-dashed and red curves indicate the velocity stability or increase with presure, respectively. Note that the hysteresis loop is not perfect between loading and unloading cycle. 

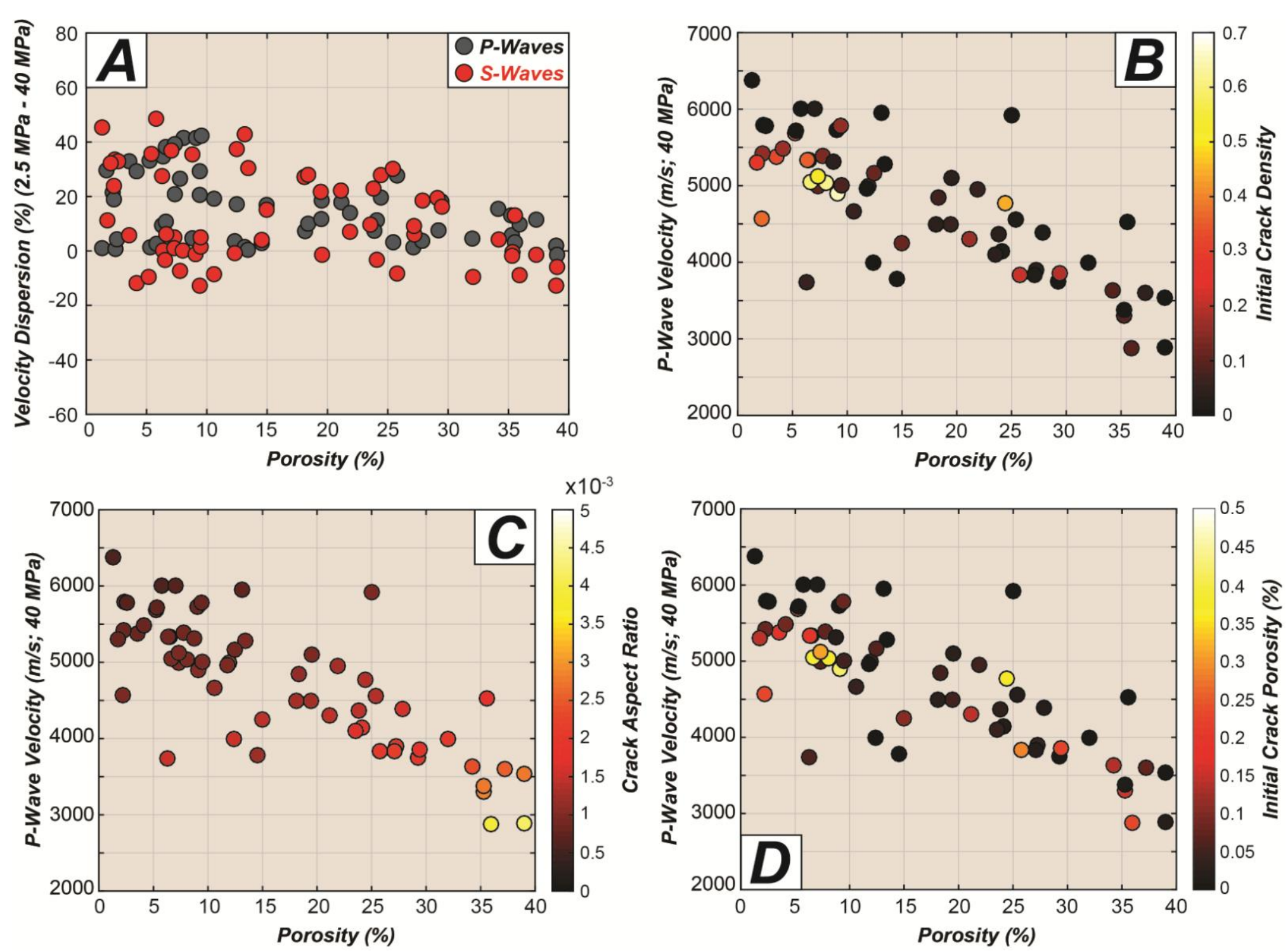

Figure 9 : A - Evolution of normalized P \& S-wave velocity dispersion with porosity at $40 \mathrm{MPa}$. B - P-wave velocity evolution with porosity. Data are discriminated with crack density value inverted from velocities between 2.5 and $40 \mathrm{MPa}$. C - P-wave velocity evolution with porosity. Data are discriminated with crack aspect ratio value closed at $40 \mathrm{MPa}$. D - P-wave velocity evolution with porosity. Data are discriminated with initial crack porosity value. 


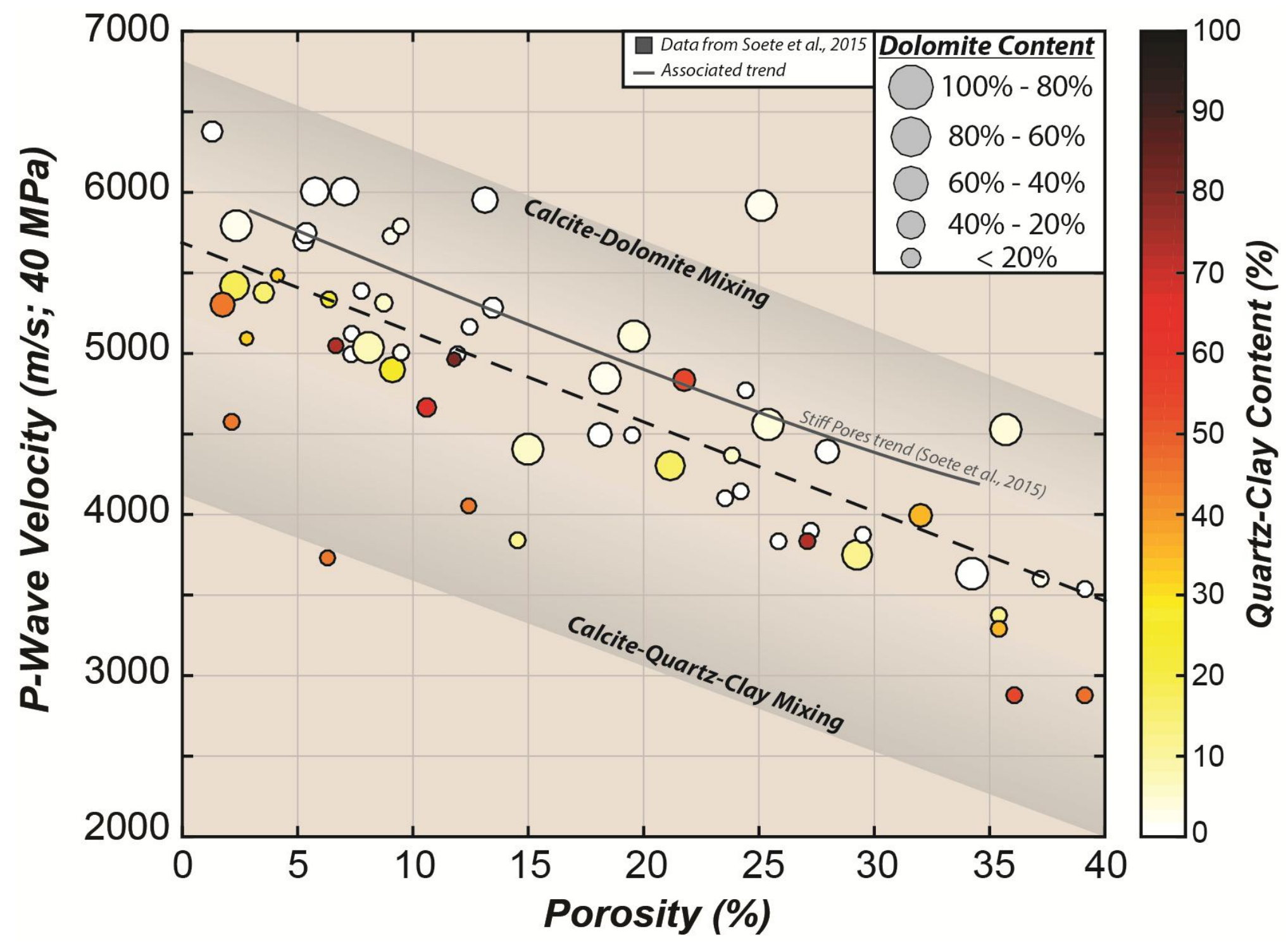

Figure 11 : P-wave velocity evolution through porosity variation. Data points are discriminated with their quartz/clay content (dot color, associated with the side colobar), and with their dolomite content (dot size). Two mineralogical domains are individualized (calcite-dolomite mixing and calcite-quartz mixing zones). Evolution trend collected from Soete et al., 2015 showing velocity-porosity relationship where stiff pore inclusions are dominant is also reported in the figure. 

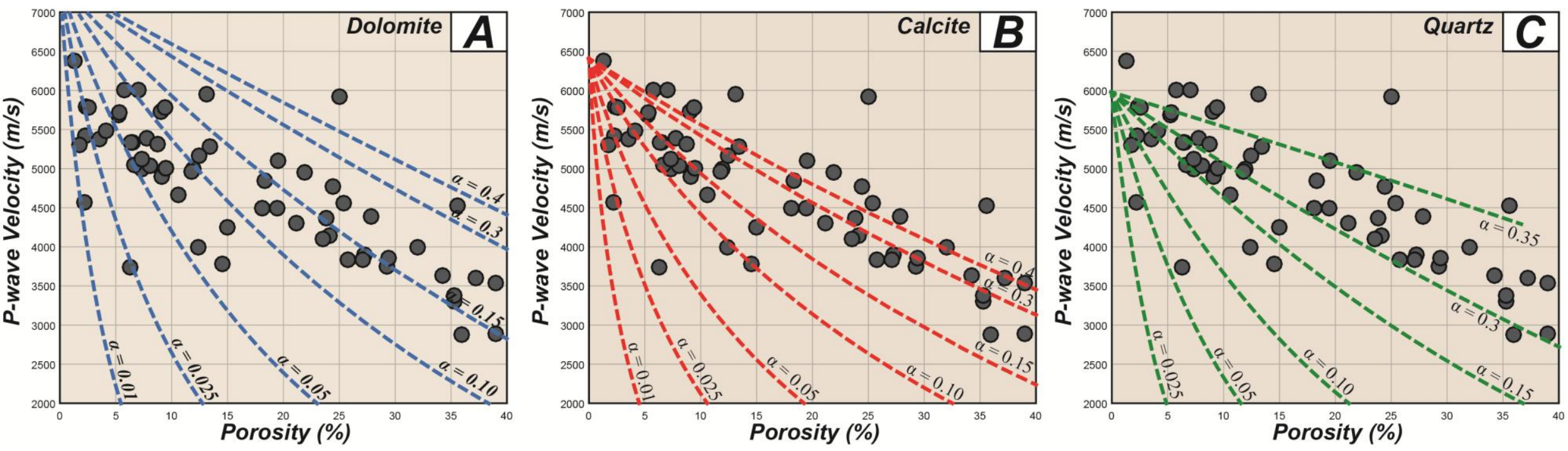

Figure 12 : Analytical simulation of P-wave velocity using the crack-like pores model from David et al., 2011. Simulations are done on three different matrix mineralogies (A - dolomite, B - calcite and C quartz) for a wide range of aspect ratio values from $\alpha=10^{-2}$ to $\alpha=0.34$. 


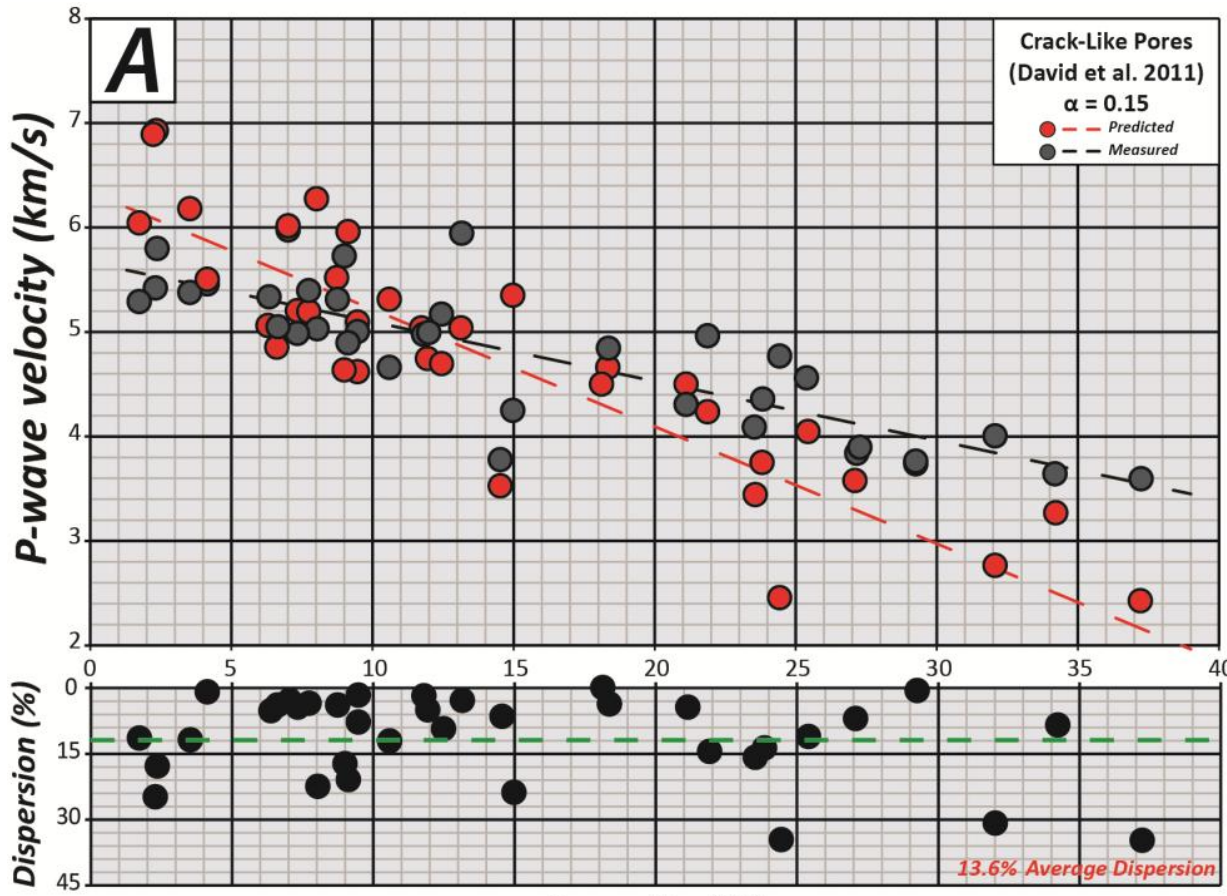

Porosity (\%)

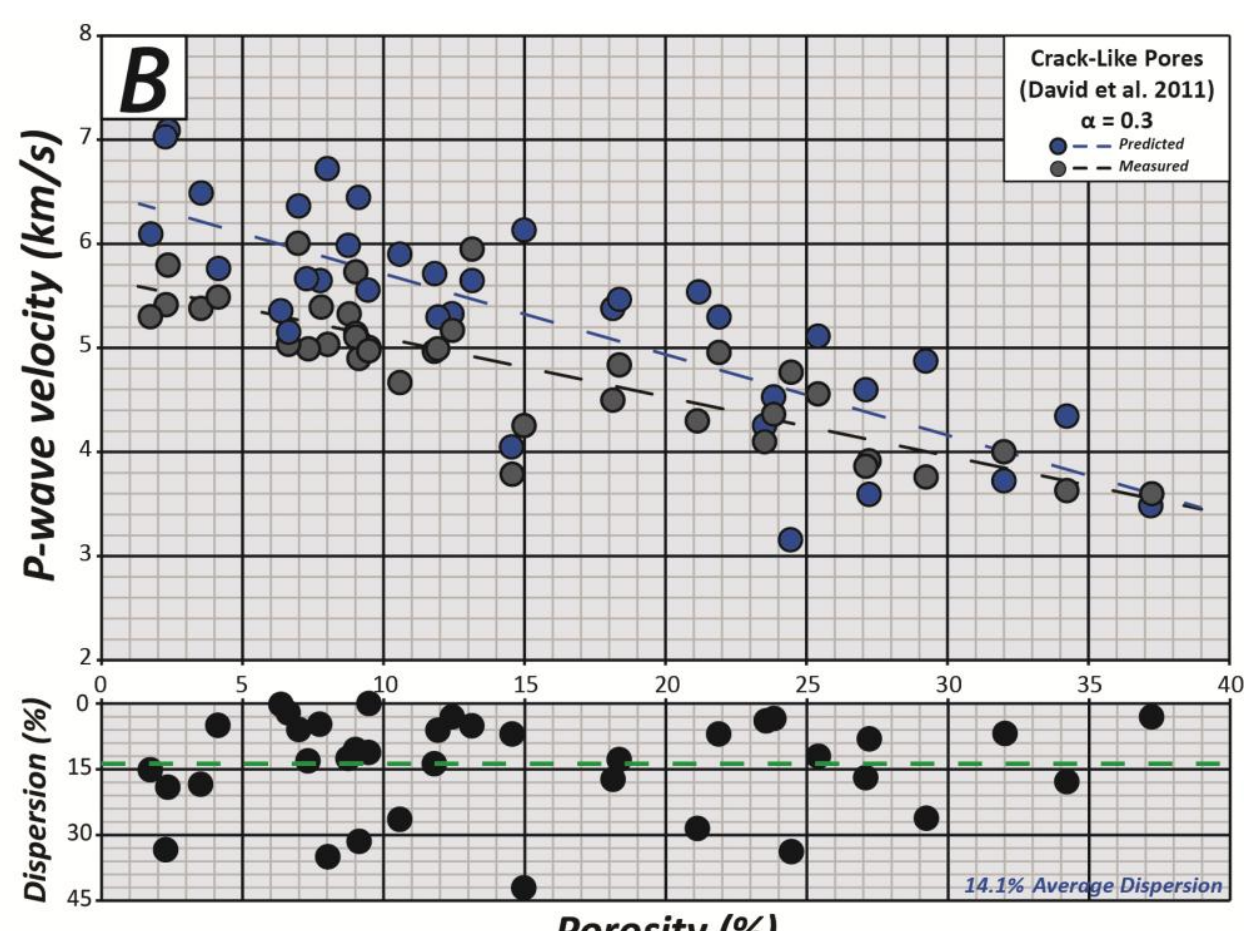

Porosity (\%)

Figure 13 : Analytical simulation of P-wave velocity for two fixed aspect ratio values $(A-\alpha=0.15$ and $B-\alpha$ $=0.3$ ). Simulations take into account the porosity and the mean mineralogy inferred from XRD analysis, for each sample. Dispersion is the normalized difference between prediction and measurements. 


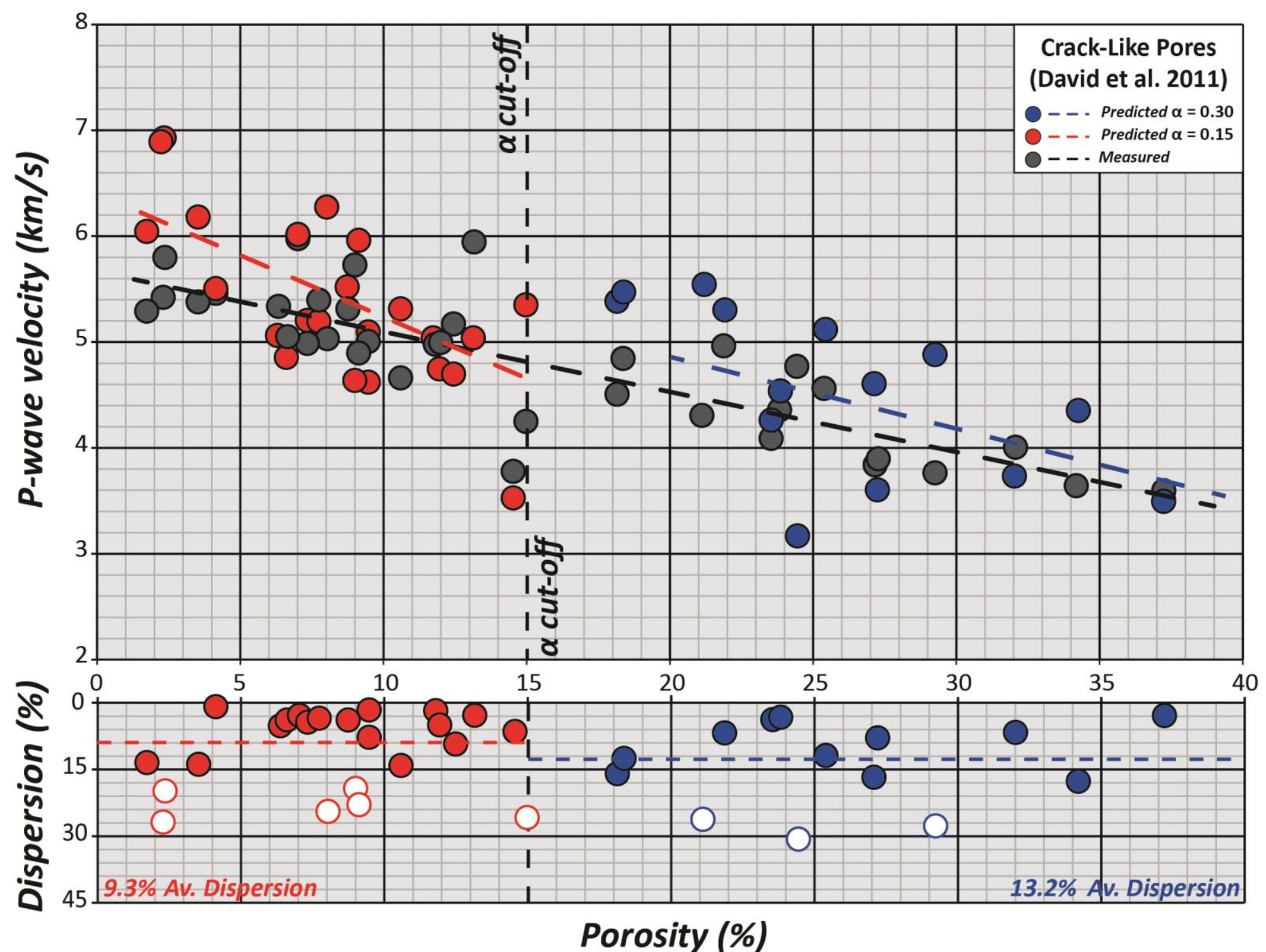

Figure 14 : Analytical simulation of P-wave velocity for two fixed aspect ratio values ( $\alpha=0.15$ between $0 \%$ and $15 \%$ porosity, and $\alpha=0.3$ beyond $15 \%$ ). Simulations take into account the porosity and the mean mineralogy inferred from XRD analysis, for each sample. Dispersion is the normalized difference between prediction and measurements. White dots are sample characterized by buggy and " rounded " biomoldic porosity. 
